\title{
Left coronary arteriovenous malformation with fistulous connections to the lefit and right ventricles
}

\section{Elzbieta Suchon, Magdalena Kostkiewicz, Wojciech Szot} Nuclear Medicine Dept, John Paul II Hospital, Krakow, Poland

[Received 1 || 2012; Accepted 16 || 2012]

\section{Abstract}

A 20-year-old man with right bundle branch block in recorded ECG was referred to our department. His physical examination was unremarkable. Transthoracic echocardiography showed a severe hypertrophy of the interventricular septum (22 $\mathrm{mm}$ ) which contained multiple echo-free spaces of the vascular nature. A flow pattern suggestive to a coronary artery fistula into the left ventricle was recorded. The patient was referred for a coronary angiography, which revealed an arteriovenous malformation starting from the septal branch of the enlarged left anterior descending artery. The malformation communicated with the lumen of the left (arterial phase) and right ventricle (venous phase) as well. The posterior descending artery was fed exclusively from the described arteriovenous malformation. ${ }_{99 m}$ Tc MIBI SPECT images showed a moderately reversible perfusion defect in the inferior wall, suggesting non-critical ischemia of this region.

Key words: coronary artery fistula, transthoracic echocardiography, ${ }^{99 m} \mathrm{Tc}$ MIBI SPECT

Nuclear Med Rev 2012; 15, 1: 80-82

Correspondence to: Magdalena Kostkiewicz MD, PhD Nuclear Medicine Dept, John Paul II Hospital

Pradnicka 80 Str., 31-202 Krakow, Poland
A 20-year-old man with RBBB on ECG was referred to our department. His physical exam was unremarkable. Transthoracic echocardiography (TTE) showed a severe hypertrophy of the interventricular septum (22 $\mathrm{mm}$ ) which contained multiple echo-free spaces which were filled with color when using the Doppler method, indicating the vascular nature of the lesion (Figure 1). Moreover, a flow pattern suggestive of a coronary artery fistula into the left ventricle was recorded (Figure 2). Accordingly, the patient was referred for a coronary angiography, which revealed

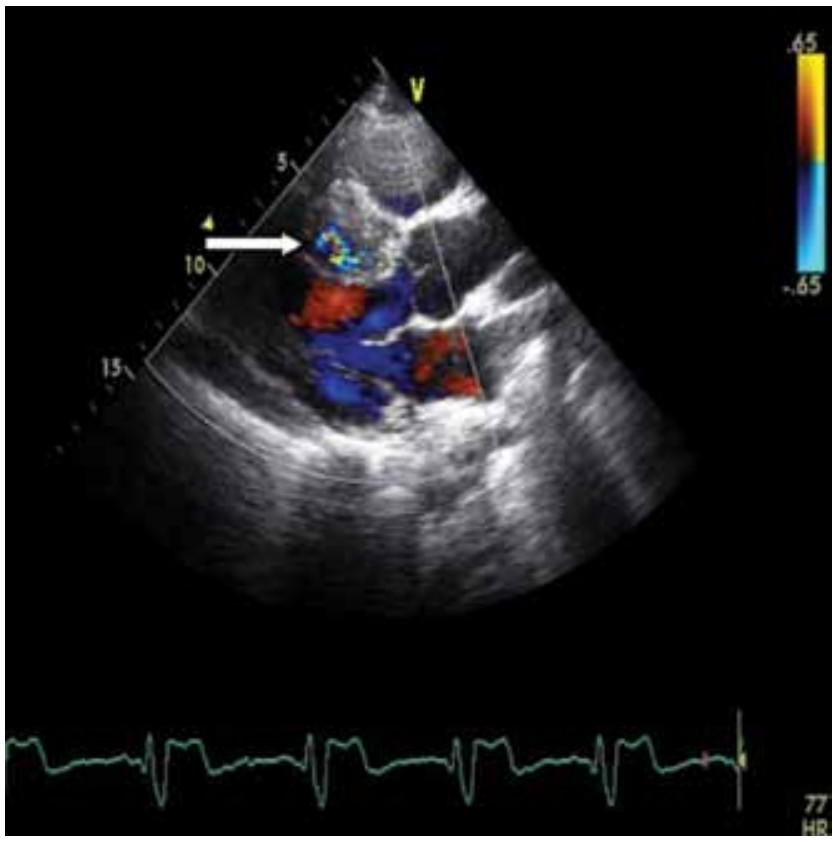

Figure 1. Transthoracic echocardiography (long axis view) demonstrates a severe hypertrophy of interventricular septum with vascular structures seen in color Doppler (arrow) 


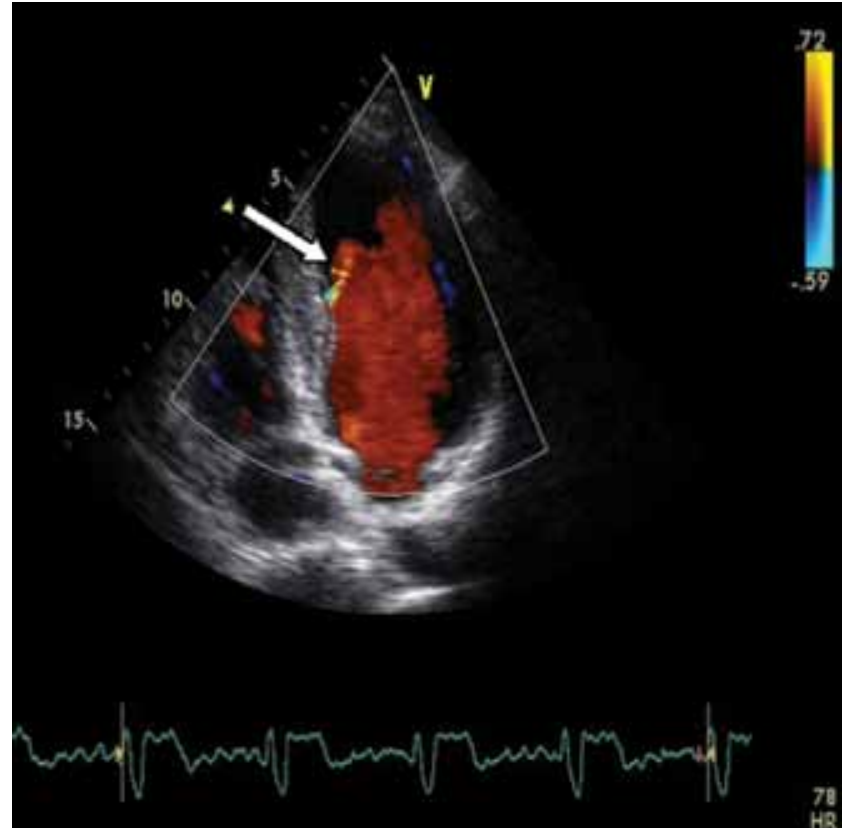

Figure 2. Transthoracic color-flow Doppler echocardiography (apical 4-chamber view) shows a fistula draining into the left ventricle (arrow)

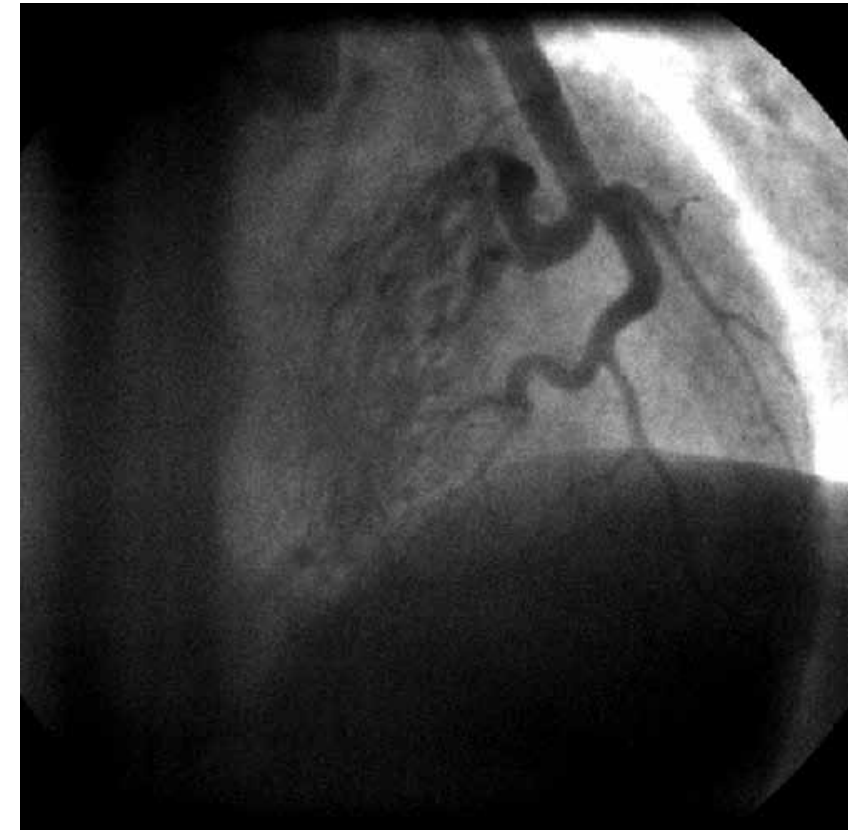

Figure 3. Coronary angiography demonstrates an arteriovenous malformation (arrow) starting from the septal branch of the enlarged left anterior descending artery

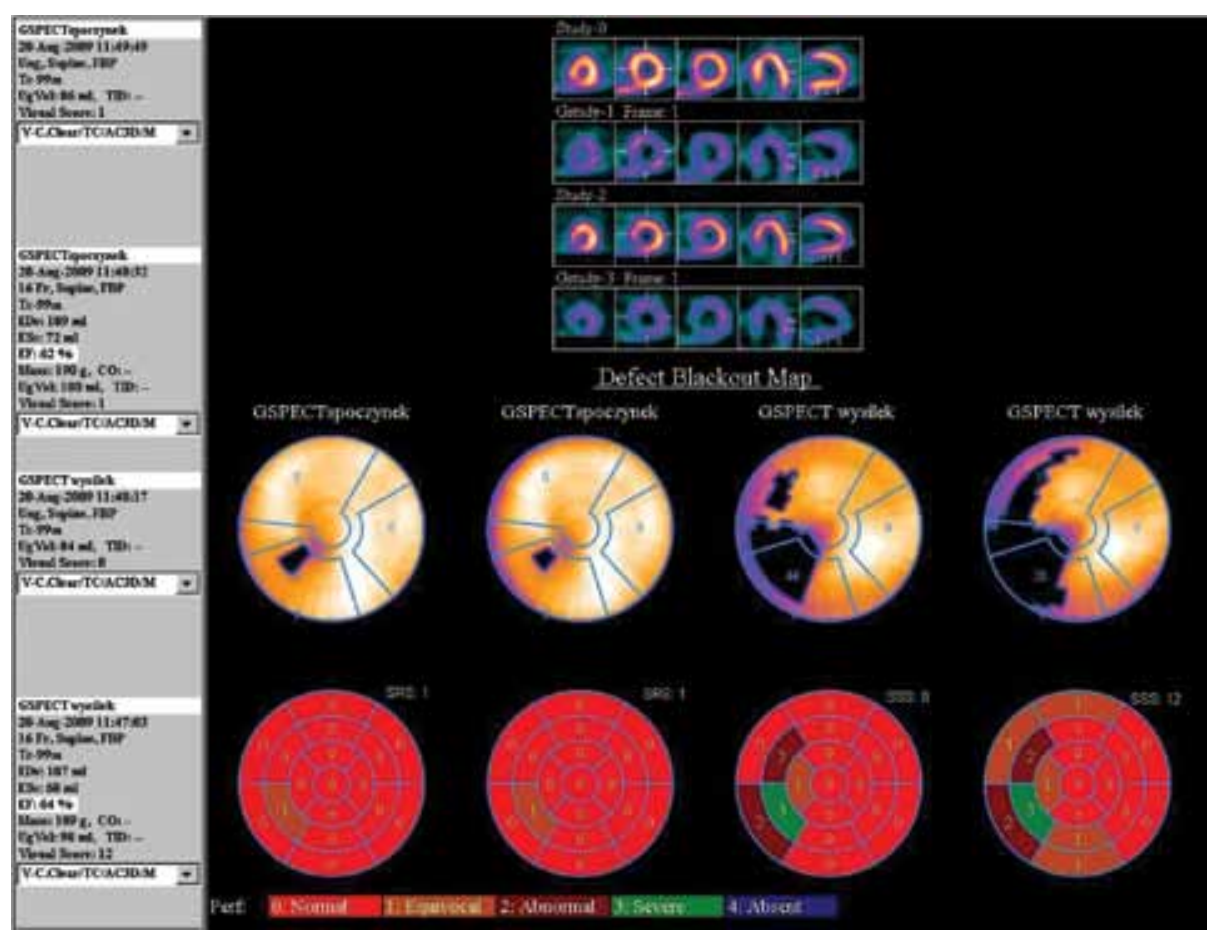

Figure 4. Comparison of myocardial perfusion in GSPECT study at rest and stress. Please note the extent of perfusion defect at stress in the area of infero-septal wall with lower grade extent to antero-septal wall (SRS: Summed Rest Score, SSS: Summed Stress Score)

an arteriovenous malformation starting from the septal branch of the enlarged left anterior descending artery (Figure 3). The malformation communicated with the lumen of the left (arterial phase) and right ventricle (venous phase) as well. The posterior descending artery (PDA) was fed exclusively from the described arteriovenous malformation.
The treadmill protocol was clinically negative, but a horizontal ST segment depression of $1.5 \mathrm{mV}$ developed in multiple ECG derivation. ${ }^{99 m} \mathrm{mC}$ MIBI SPECT images acquired 30 min after peak exercise and 60 min after rest injections demonstrated a moderately reversible perfusion defect in the inferior wall, suggesting non-critical ischemia of this region (Figures 4, 5). 


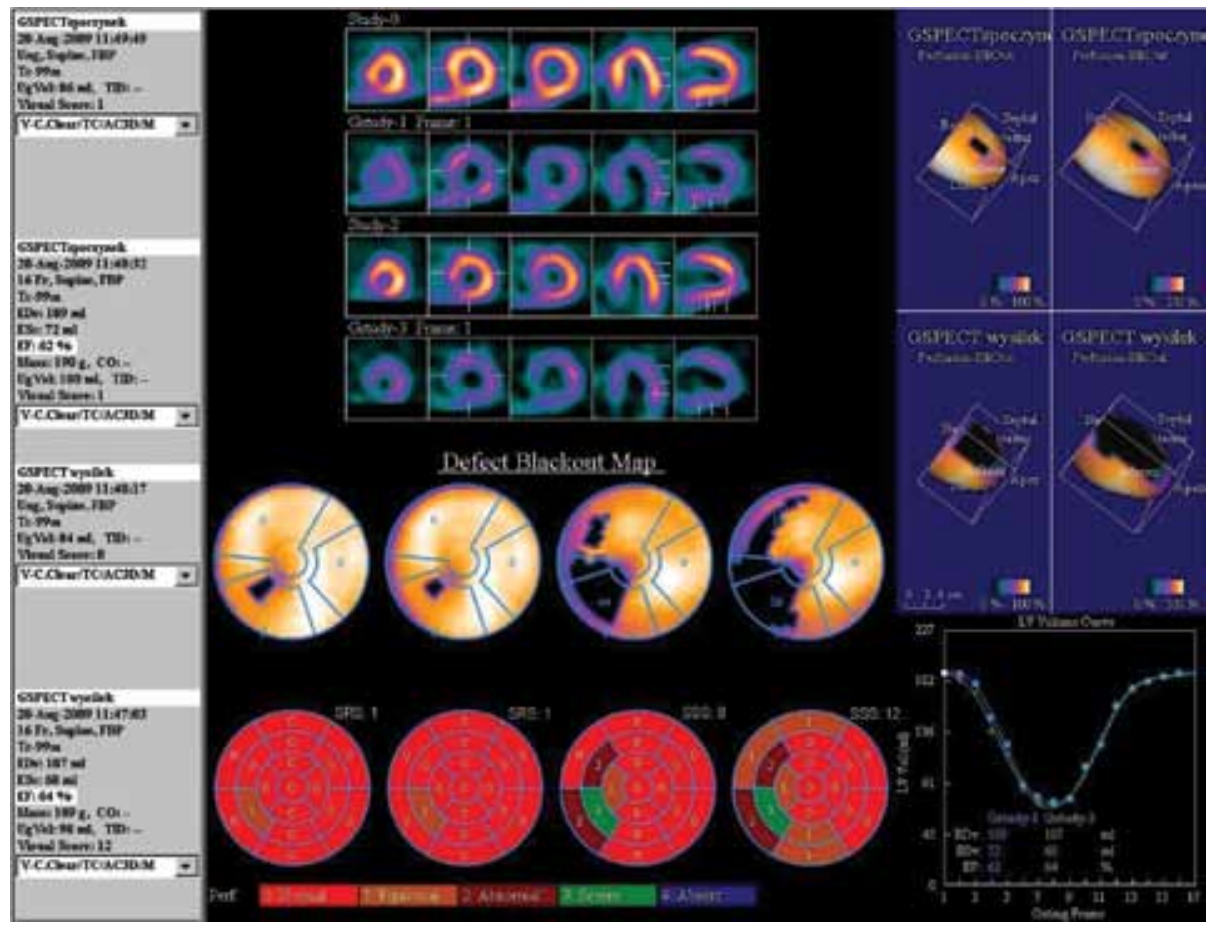

Figure 5. Left ventricular function and perfusion in GSPECT study at rest and stress. Please note a slight improvement in left ventricular function at stress, possible a result of hyper contractility of anterior and latero-inferior wall

The imaging data was crucial in deciding to keep the patient under observation despite favorable clinical data.

Coronary artery fistulas vary widely in their morphological appearance and presentations. The majority of patients are asymptomatic but angina, myocardial infarction, heart failure and endocarditis have been reported [1, 2]

Therapeutic approaches for coronary artery fistula are generally controversial. Options include percutaneous intracoils placement, surgical intervention or conservative treatment [1-4]. In our patient, the fistulae were small, asymptomatic and not suitable for percutaneous closure. We decide to treat the patient conservatively. The accompanying images underscore the importance of using different methods like TTE, SPECT and coronary angiography to most effectively diagnose patients with coronary artery malformations, as in this case.

\section{References}

1. Palloshi A, Aprigliano G. Myocardial ischemia due to a large coronary-pulmonary fistula with plexus-like morphology. J Cardiovasc Med (Hagerstown) 2008; 9: 1163-1165.

2. Sato F, Koishizawa T. Stress/Rest (99m) Tc-MIBI SPECT and 123I -BMIPP scintigraphy for indication of surgery with coronary artery to pulmonary artery fistula. Int Heart J 2005; 46: 355-361.

3. Cebi N, Schulze-Waltrup N, Frömke J, Scheffold T, Heuer H. Congenital coronary artery fistulas in adults: concomitant pathologies and treatment. Int J Cardiovasc Imaging 2008; 24: 349-355.

4. Arslan S, Gurlertop Y, Elbey MA, Karakelleoglu S. Multiple coronary-cameral fistulae causing angina pectoris. Tex Heart Inst J 2009; 36: 622-623. 